\title{
Concordance between the estimates of wasting measured by weight-for-height and by mid-upper arm circumference for classification of severity of nutrition crisis: analysis of population-representative surveys from humanitarian settings
}

Oleg Bilukha and Eva Leidman *iD

\begin{abstract}
Background: Despite frequent use of mid-upper arm circumference (MUAC) to assess populations at risk of nutrition emergencies, as well as evidence that measurement of children based on MUAC identifies different children than weight-for-height (WHZ) as wasted, no crisis classification thresholds based on prevalence of wasting by MUAC currently exist.

Methods: We analyzed 733 population-representative anthropometric surveys from 41 countries conducted by Action Contre la Faim (ACF) and the United Nations High Commissioner for Refugees (UNHCR) between 2001 and 2016. Children aged 6-59 months were classified as wasted if they had a WHZ $<-2$ and/or a MUAC $<125 \mathrm{~mm}$. Prevalence of wasting as assessed by $\mathrm{WHZ}$ and by MUAC were compared using correlations and linear regression models adjusting for stunting prevalence, sex and age distribution of the sample. Median prevalence of wasting by MUAC corresponding to each of the WHZ-based crisis thresholds was examined.

Results: Median prevalence of wasting by WHZ was 10.47\% (IQR: 6.34-17.55\%) and by MUAC was 6.66\% (IQR:4.12-10.88\%). Prevalence of wasting by WHZ exceeded prevalence by MUAC in 543 (74.1\%) surveys and median prevalence by WHZ was greater in 30 (73.17\%) countries. Prevalence of wasting by WHZ is poorly correlated with prevalence of wasting by MUAC $(\rho=0.55)$. $R^{2}$ was 0.36 for unadjusted and 0.45 for adjusted linear regression model. The difference between the prevalence by WHZ and by MUAC increased as the overall prevalence by WHZ increased $(\rho=0.69)$. Surveys with prevalence of wasting by WHZ approximately equal to thresholds for "poor" (5\% $\pm 2.5 \%)$, "serious" (10\% $\pm 2.5 \%)$, "emergency" (15\% $\pm 2.5 \%)$, and "famine" (30\% $\pm 2.5 \%)$ were observed to have median prevalence of wasting by MUAC of 4.51\% (IQR: 2.73-6.81\%), 6.67\% (IQR: 4.27-10.03\%), 8.15\% (IQR: $5.11-11.86 \%)$, and 15.71\% (IQR: 10.28-17.50\%), respectively. There was a very substantial overlap of MUAC values across the threshold categories.

Conclusions: Given a poor correlation between population prevalence of wasting by WHZ and by MUAC, classification of surveys based on prevalence of wasting by MUAC will result in poor concordance with current WHZ-based crisis thresholds, even if regional differences are considered, regardless of the cutoffs used.
\end{abstract}

Keywords: Wasting, Survey, Nutrition, Humanitarian

\footnotetext{
* Correspondence: eleidman@cdc.gov

Emergency Response and Recovery Branch, Division of Global Health

Protection, Center for Global Health, Centers for Disease Control, 1600 Clifton

Road, Atlanta, GA 30329, USA
}

(c) The Author(s). 2018 Open Access This article is distributed under the terms of the Creative Commons Attribution 4.0 International License (http://creativecommons.org/licenses/by/4.0/), which permits unrestricted use, distribution, and reproduction in any medium, provided you give appropriate credit to the original author(s) and the source, provide a link to the Creative Commons license, and indicate if changes were made. The Creative Commons Public Domain Dedication waiver (http://creativecommons.org/publicdomain/zero/1.0/) applies to the data made available in this article, unless otherwise stated. 


\section{Background}

Prevalence of acute malnutrition is commonly used to benchmark the severity of a nutritional emergency to help inform the scale and scope of humanitarian response activities. Prevalence in a given context is compared with global standard thresholds. The World Health Organization (WHO) initially outlined guidance on these standard thresholds in 1995, modifying guidance from a 1992 consultation by the WHO Eastern Mediterranean regional office [1]. The guidance proposed classification of a situation using thresholds of less than 5\% prevalence of wasting ("acceptable"), less than 10\% ("poor"), less than $15 \%$ ("serious") and equal to or greater than $15 \%$ ("critical"). The Management of Nutrition in Major Emergencies, a joint guidance document drafted in 2000 by WHO, United Nations High Commissioner for Refugees (UNHCR), International Federation of Red Cross (IFRC), and World Food Programme (WFP), included these same thresholds prompting a more universal adoption [2]. In 2004, the need for a higher, famine threshold was proposed by Howe and Devereux [3]. Currently, both the Integrated Phase Classification (IPC) used in East Africa and Asia and the Cadre Harmonisé $(\mathrm{CH})$ used in the Sahel and West Africa use a cutoff of 30\% prevalence of wasting as a threshold for famine, such that prevalence of wasting is used to classify a situation as Phase I $(<5 \%)$, Phase $2(5-<10 \%)$, Phase $3(10-<15 \%)$, Phase $4(15-<30 \%)$ or Phase $5(\geq 30 \%)[4,5]$.

The above standard thresholds are all based on prevalence of wasting as assessed by weight-for-height $\mathrm{Z}$ scores (WHZ) [1, 2, 4, 5]. In addition to WHZ, wasting can be assessed using mid-upper arm circumference (MUAC). Since in 2005 WHO, WFP, United Nations Children's Fund (UNICEF), and the Standing Committee on Nutrition (SCN) recommended MUAC as independent measure of wasting used as a criterion for admission into selective nutrition feeding programs [6-8]. However, separate thresholds for classifying a crisis based on prevalence of wasting as assessed by MUAC do not exist. Previous research demonstrating substantial discrepancy in diagnosis of children as wasted using WHZ and MUAC has prompted questions about the validity of applying WHZ-based thresholds to estimates of wasting based on MUAC. Based on an analysis of over 560 surveys from 31 countries, WHO estimated that only about 4 in 10 children were identified as wasted by both WHZ and MUAC, concluding that "the cases selected using weight-for-height and MUAC were not the same" [6]. Multi-country analysis by Grellety et al. using 1832 surveys from 47 countries similarly highlighted that a large proportion of children were identified as wasted by MUAC but not WHZ and by WHZ but not MUAC, adding that the proportion of children in each of these categories varied widely by country [9]. Analysis by
Roberfroid et al. found that stunting, sex, and age all influenced diagnosis of acute malnutrition by MUAC but not WHZ [10]. However, while there is evidence to suggest poor correlation between WHZ and MUAC diagnosis of individual children, whether or not this translates into population level differences in prevalence of wasting by WHZ and MUAC has yet to be evaluated.

Mid-upper arm circumference is increasingly used to measure wasting, especially as part of community-based screenings and at remote clinics where height boards and other anthropometric equipment may not be available. Additionally, several studies suggest that low MUAC better predicts mortality than low WHZ, as summarized by Briend et al [11]. MUAC only assessments are particularly common in humanitarian settings with extreme insecurity. In the absence of clear guidance on thresholds for classifying prevalence of acute malnutrition by MUAC, the WHZ-based thresholds have been applied in many contexts. The Cadre Harmonisé, for example, recommends this approach for the Sahel and West Africa [4]. The Integrated Food Security and Nutrition Phase Classification technical committee has identified the need for secondary analysis of existing survey data to explore the possibility of deriving the thresholds for classifying severity of wasting at the population level using prevalence of wasting by MUAC where WHZ based anthropometry data are not available.

The objective of this research therefore was to explore the concordance of the prevalence of wasting by WHZ and MUAC at the population level and the possibility of deriving MUAC-based crisis thresholds corresponding to the existing WHZ-based thresholds. The focus of the analysis was on total rather than severe wasting, as WHO-recommended emergency thresholds are based on the prevalence of total wasting $[1,2]$. To this aim, we assessed the correlation of prevalence of low MUAC and low WHZ in survey samples globally, as well as described prevalence of wasting by MUAC in populations with prevalence approximately equal to poor, serious, critical, and famine thresholds as determined by WHZ.

\section{Methods}

Data included in these analyses were from small-scale field nutrition surveys conducted in humanitarian settings by Action Contre la Faim (ACF) International (an international humanitarian non-governmental organization focused on nutrition in humanitarian settings worldwide) and by the United Nations High Commissioner for Refugees (UNHCR). Data were drawn from a database of 808 population-representative cross sectional surveys conducted between 2001 and 2016 [12, 13]. Surveys with sample sizes smaller than 196 persons and cluster surveys with fewer than 25 clusters were excluded a priori from all analyses as they did not meet minimum standards for 
small-scale cluster surveys [9]. Surveys that did not collect both MUAC and weight-for-height (weight, height, age and sex) were also excluded.

Weight-for-Height Z-scores (WHZ) were calculated for each child using the WHO 2006 growth standards using the WHO SAS macro [14]. Only children aged 6-59 months were included in the analyses. Prevalence of wasting by WHZ for each survey reflects the proportion of children with WHZ less than -2. Outlier observations were excluded from a survey if Z-score of a child fell outside the flexible exclusion range of \pm 4 Z-scores from the observed survey sample mean, as described by WHO [1]. Prevalence of wasting by MUAC for each survey reflects the proportion of children with MUAC values less than $125 \mathrm{~mm}$. MUAC values less than $70 \mathrm{~mm}$ and greater than $220 \mathrm{~mm}$ were excluded as outliers. Individual observations within each survey were also excluded from calculations of wasting by WHZ for children without information on height, weight, age or sex and from calculations of wasting by MUAC for children without information on MUAC and age. Cases of bilateral pitting edema were not included in estimated prevalence of wasting by WHZ or MUAC; edema cases were relatively rare in all surveys, representing approximately 3 per 1000 (mean: 0.32\%) sampled children.

Countries where the surveys were conducted were categorized into seven geographical or country groupings (Latin America and the Caribbean; Eastern and Southern Africa; Democratic Republic of Congo (DRC); West and Central Africa; South, Southeast Asia and Pacific; Sudan; Middle East and North Africa) as seen in Table 1. DRC and Sudan were analyzed as its own grouping given the large number of surveys conducted in both countries.

The first aim of the analysis was to describe the relationship between prevalence of wasting as assessed by WHZ and the prevalence of wasting as assessed by MUAC on the same population. Spearman correlations were therefore calculated to describe the correlation between prevalence of wasting by WHZ and by MUAC, as well as between the difference in prevalence by WHZ and MUAC and the prevalence by each WHZ and MUAC survey. A multivariate model was then constructed to explore the relationship of the prevalence by WHZ and by MUAC, controlling for key factors shown in previous research to be associated with the prevalence of wasting by MUAC. These factors included stunting prevalence, sex distribution, and age distribution of the survey sample [10]. Prevalence of wasting by MUAC as an outcome and all predictor variables were modeled as continuous linear terms. Sex ratio was calculated as the proportion of females in the survey sample. Age ratio was calculated as the proportion of younger children aged 6-29 months in the survey sample. Observations with significantly high leverage or Cook's distance were removed from the multivariable analyses. The regression analysis was repeated using logit-transformed independent and dependent variables [15]. To assess the reproducibility of the results, analysis above was repeated with DRC included in the West and Central Africa region and Sudan included in the Middle East North Africa, and separately for surveys conducted by ACF and UNHCR.

Second, we described the prevalence of wasting by MUAC in surveys with the prevalence of wasting by WHZ corresponding to the existing WHZ-based crisis thresholds $(5,10,15$ and $30 \%)$ to assess the feasibility of deriving corresponding thresholds using MUAC. Surveys with prevalence of wasting by WHZ within $\pm 2.5 \%$ of the $5,10,15$, and $30 \%$ crisis thresholds were included in the analysis. For example, to explore prevalence of MUAC that may correspond to the 10\% WHZ-based crisis threshold we used surveys with a prevalence of wasting by WHZ between 7.5 and $12.5 \%$. Median and interquartile range (IQR) for prevalence of wasting by MUAC were calculated for each of the sub-sets of surveys with prevalence approximately equal to the four thresholds, overall and by geographic region. The analysis was repeated using only the surveys within $\pm 1.5 \%$ of the crisis thresholds.

Finally, we explored concordance of the possible MUAC classification by determining the proportion of surveys that would be classified into the same crisis category if categorized separately based on prevalence of wasting by WHZ and by MUAC. An example set of MUAC thresholds was used for this classification, derived from the observed median values observed in the previous stage analysis.

All data were aggregated and cleaned using SAS Version 9.3, analysis was performed in Stata IC Version 14.2, and figures were produced in JMP Version 13.0.0.

\section{Results}

In total, 808 surveys were reviewed for this study. Seventy-five surveys were excluded from the analysis: 60 surveys did not collect MUAC measurements and another 15 had fewer than 25 clusters and/or had a sample size smaller than 196 children, resulting in 733 surveys from 41 countries retained for analysis. As seen in Table 1 , the countries with the largest number of surveys were Sudan (150 surveys), DRC (130), Chad (67), Ethiopia (59) and Kenya (46). All other countries had 32 of fewer surveys each. Among selected surveys, $0.7 \%$ of children aged 6-59 months were excluded due to missing anthropometric values (sex, weight, height or MUAC) and an additional $0.4 \%$ were excluded due to out of range values for WHZ or MUAC. After exclusions, these surveys represent data from approximately 550 thousand children. 
Table 1 Number of included surveys, children and prevalence of wasting by country and region, 2001-2016

\begin{tabular}{|c|c|c|c|c|}
\hline & Number of surveys & Number of children & $\begin{array}{l}\text { Prevalence of wasting } \\
\text { by WHZ Median (IQR) }\end{array}$ & $\begin{array}{l}\text { Prevalence of wasting } \\
\text { by MUAC Median (IQR) }\end{array}$ \\
\hline Latin America and the Caribbean & 16 & 11,808 & $3.95 \%(2.87-4.46)$ & $2.99 \%(2.17-4.35)$ \\
\hline Bolivia & 1 & 884 & $1.28 \%$ & $0.35 \%$ \\
\hline Guatemala & 2 & 1432 & $2.10 \%(0.39-3.80)$ & $3.83 \%(0.20-7.46)$ \\
\hline Haiti & 13 & 9492 & $4.27 \%(3.81-4.55)$ & $3.00 \%(2.77-4.15)$ \\
\hline Eastern and Southern Africa & 190 & 128,404 & $9.15 \%(5.76-17.88)$ & $4.66 \%(2.91-7.49)$ \\
\hline Angola & 1 & 870 & $5.77 \%$ & $4.53 \%$ \\
\hline Botswana & 1 & 215 & $3.66 \%$ & $0.61 \%$ \\
\hline Burundi & 7 & 3493 & $5.76 \%(4.23-7.08)$ & $3.03 \%(2.08-9.33)$ \\
\hline Eritrea & 2 & 859 & $20.22 \%(18.89-21.55)$ & $4.31 \%(4.02-4.60)$ \\
\hline Ethiopia & 59 & 29,554 & $16.93 \%(8.94-21.77)$ & $4.95 \%(3.59-8.50)$ \\
\hline Kenya & 46 & 30,173 & $10.92 \%(7.88-15.73)$ & $3.74 \%(2.66-5.28)$ \\
\hline Madagascar & 1 & 635 & $7.47 \%$ & $6.45 \%$ \\
\hline Mozambique & 1 & 406 & $3.26 \%$ & $3.26 \%$ \\
\hline Rwanda & 13 & 4694 & $4.74 \%(3.85-5.97)$ & $1.98 \%(1.82-3.11)$ \\
\hline Somalia & 3 & 2688 & $15.85 \%(15.10-37.79)$ & $5.70 \%(2.22-25.35)$ \\
\hline South Sudan & 17 & 15,518 & $11.4 \%(8.19-22.15)$ & $7.55 \%(6.17-9.84)$ \\
\hline Tanzania & 5 & 2487 & $1.90 \%(1.54-2.61)$ & $1.18 \%(0.74-3.33)$ \\
\hline Uganda & 32 & 36,022 & $4.93 \%(3.76-8.92)$ & $6.78 \%(4.34-9.08)$ \\
\hline Zambia & 2 & 790 & $4.40 \%(4.38-4.42)$ & $6.38 \%(2.35-10.40)$ \\
\hline Democratic Republic of Congo & 130 & 118,871 & $7.40 \%(4.36-10.46)$ & $7.97 \%(5.36-12.51)$ \\
\hline West and Central Africa & 146 & 90,857 & $9.60 \%(6.31-14.99)$ & $5.89 \%(3.00-9.69)$ \\
\hline Burkina Faso & 11 & 5593 & $12.41 \%(6.21-14.99)$ & $6.11 \%(2.51-8.78)$ \\
\hline Cameroon & 12 & 5614 & $9.24 \%(7.33-12.28)$ & $6.86 \%(4.65-7.92)$ \\
\hline Central African Republic & 8 & 6357 & $5.75 \%(5.40-6.63)$ & $8.02 \%(6.78-9.04)$ \\
\hline Chad & 67 & 39,428 & $9.66 \%(6.34-18.89)$ & $3.76 \%(2.06-11.09)$ \\
\hline Guinea & 5 & 4054 & $6.01 \%(5.16-7.81)$ & $3.67 \%(2.88-4.80)$ \\
\hline Liberia & 9 & 4518 & $3.57 \%(3.13-4.39)$ & $4.00 \%(1.98-5.43)$ \\
\hline Mali & 8 & 6342 & $10.74 \%(9.20-16.41)$ & $6.29 \%(5.71-7.33)$ \\
\hline Mauritania & 6 & 3693 & $12.61 \%(8.77-14.81)$ & $5.36 \%(4.14-7.90)$ \\
\hline Niger & 14 & 9825 & 13.08\% (11.07-15.95) & $8.58 \%$ (6.53-13.32) \\
\hline Sierra Leone & 6 & 5433 & $7.01 \%(6.15-7.13)$ & $9.89 \%$ (5.88-10.67) \\
\hline South East Asia and Pacific & 87 & 63,411 & $11.95 \%(7.36-17.20)$ & $8.00 \%(4.54-13.28)$ \\
\hline Afghanistan & 24 & 21,220 & $8.57 \%(5.90-11.41)$ & $9.06 \%(6.46-14.38)$ \\
\hline Bangladesh & 22 & 11,373 & $12.69 \%(9.70-14.25)$ & $5.35 \%(4.28-6.74)$ \\
\hline India & 1 & 465 & $20.58 \%$ & $10.07 \%$ \\
\hline Myanmar & 13 & 9993 & $18.26 \%(5.48-20.75)$ & $11.64 \%$ (9.66-15.05) \\
\hline Nepal & 9 & 5946 & $14.31 \%(12.50-19.58)$ & $10.55 \%(6.49-15.32)$ \\
\hline Pakistan & 13 & 10,887 & $18.45 \%(11.40-19.55)$ & $9.76 \%(6.92-14.79)$ \\
\hline Philippines & 4 & 2630 & $6.06 \%(4.77-8.64)$ & $1.21 \%(0.88-1.42)$ \\
\hline Tajikistan & 1 & 897 & $9.81 \%$ & $16.01 \%$ \\
\hline
\end{tabular}


Table 1 Number of included surveys, children and prevalence of wasting by country and region, 2001-2016 (Continued)

\begin{tabular}{lllll}
\hline & Number of surveys & Number of children & $\begin{array}{l}\text { Prevalence of wasting } \\
\text { by WHZ Median (IQR) }\end{array}$ & $\begin{array}{l}\text { Prevalence of wasting } \\
\text { by MUAC Median (IQR) }\end{array}$ \\
\hline Sudan & 150 & 130,735 & $18.96 \%(14.06-23.82)$ & $10.21 \%(7.05-14.26)$ \\
Middle East North Africa & 14 & 5602 & $6.69 \%(2.28-12.02)$ & $3.46 \%(1.23-5.75)$ \\
$\quad \begin{array}{l}\text { Djibouti } \\
\text { Iraq }\end{array}$ & 6 & 2190 & $11.42 \%(10.39-13.84)$ & $5.20 \%(3.78-7.27)$ \\
Jordan & 1 & 575 & $2.28 \%$ & $3.14 \%$ \\
$\quad$ Yemen & 4 & 1610 & $1.18 \%(0.88-2.04)$ & $1.01 \%(0.69-1.15)$ \\
All Surveys & 3 & 1227 & $5.59 \%(3.61-17.17)$ & $4.14 \%(2.38-6.53)$ \\
\hline
\end{tabular}

Prevalence of wasting by WHZ was higher than by MUAC for most surveys. Median prevalence of wasting by WHZ was $10.47 \%$ (IRQ: $6.34-17.55 \%$ ) and by MUAC was $6.66 \%$ (IQR: 4.12-10.88\%) (Table 1). Prevalence of wasting by WHZ exceeded prevalence by MUAC in 543 (74.1\%) surveys and median prevalence by WHZ was greater in $30(73.17 \%)$ countries. The difference in median prevalence was greatest among surveys in East Africa such as Eritrea (15.91\%), Ethiopia (11.98\%), and Somalia (10.15\%) as well as in India (10.51\%).

The data suggest a positive but relatively weak monotonic correlation $(\rho=0.5485)$ between prevalence of wasting by WHZ and by MUAC (Table 2 and Fig. 1a). By region, correlation was highest for the Middle East and North Africa $(\rho=0.8901)$ and DRC $(\rho=0.6822)$ and lowest for Eastern and Southern Africa $(\rho=0.3553)$. Rho for all surveys was improved when prevalence of wasting by WHZ was correlated with the difference between the prevalence of wasting by WHZ and by MUAC ( $\rho=0.6859)$. Notably, difference in prevalence by WHZ and by MUAC was greatest for surveys with higher prevalence of wasting by WHZ. Conversely, overall correlation was lowest when prevalence of wasting by MUAC was correlated with the difference between the prevalence of wasting by WHZ and by MUAC $(\rho=-0.1634)$. The strength of the correlation varied by region (Table 2 and Fig. 1b, c). These correlations did not change markedly when surveys from ACF and UNHCR were analyzed separately (not presented).
$\mathrm{R}^{2}$ in the univariate linear model with prevalence of WHZ as a predictor and prevalence by MUAC as an outcome was $0.36 . \mathrm{R}^{2}$ in the multivariate model adjusted for prevalence of stunting, the proportion of younger children (aged 6-29 months of age), and the proportion of females, increased to 0.46 (Table 3). Multivariate model results suggest that a $1 \%$ increase in prevalence of wasting by WHZ was associated with a $0.5 \%$ increase in prevalence of wasting by MUAC; this association was highly significant $(p<0.001)$. All other co-variates were also positively associated with prevalence of wasting by MUAC. Prevalence of stunting and the proportion of younger children were both significant $(p<0.001$ for both), whereas the proportion of females was not $(p=0.218)$ (Table 3). Logit transformation of all variables in the model did not improve fit of either univariate or multivariate models ( $R^{2}=0.35$ and 0.43 , respectively).

Table 4 and Fig. 2 present the median prevalence of wasting by MUAC corresponding to each of the WHZ-based crisis thresholds (5, 10, 15, and 30\%). Overall, median prevalence of wasting by MUAC was 4.51\% (IQR: 2.73-6.81\%) for surveys near the "poor" threshold ( $5 \pm 2.5 \%)$, 6.67\% (IQR: 4.27-10.03\%) for surveys near the "serious" threshold $(10 \pm 2.5 \%), 8.15 \%$ (IQR: 5.11-11.86\%) for surveys near the "emergency" threshold (15 $\pm 2.5 \%)$, and 15.71\% (IQR: $10.28-17.50 \%$ ) for surveys near the "famine" threshold $(30 \pm 2.5 \%)$. Median MUAC thresholds corresponding to 5, 10, 15\%

Table 2 Spearman's correlation (rho) for prevalence of wasting by weight-for-height and by mid-upper arm circumference overall and differences in prevalence ${ }^{a}$

\begin{tabular}{llll}
\hline & WHZ v. MUAC & (WHZ- MUAC) v MUAC & $($ WHZ-MUAC) v WHZ \\
\hline Latin America and Caribbean & 0.3667 & -0.6377 & 0.3726 \\
Eastern and Southern Africa & 0.3553 & -0.1599 & $\mathbf{0 . 8 2 7 9}$ \\
Congo, DRC & $\mathbf{0 . 6 8 2 2}$ & -0.4811 & 0.2513 \\
West and Central Africa & $\mathbf{0 . 6 2 4 7}$ & -0.0886 & $\mathbf{0 . 6 6 5 2}$ \\
Sudan & $\mathbf{0 . 6 1 8 7}$ & -0.1317 & $\mathbf{0 . 6 2 1 0}$ \\
Middle East North Africa & $\mathbf{0 . 8 9 0 1}$ & $\mathbf{0 . 6 3 5 2}$ & $\mathbf{0 . 8 8 5 7}$ \\
South East Asia and Pacific & $\mathbf{0 . 5 6 3 9}$ & -0.4296 & 0.4501 \\
All Surveys & $\mathbf{0 . 5 4 8 5}$ & -0.1634 & $\mathbf{0 . 6 8 5 9}$ \\
\hline
\end{tabular}

Rho values greater than 0.5 are indicated in bold 
a

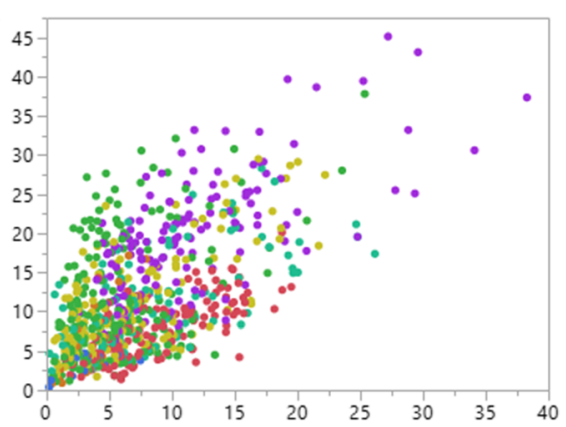

b

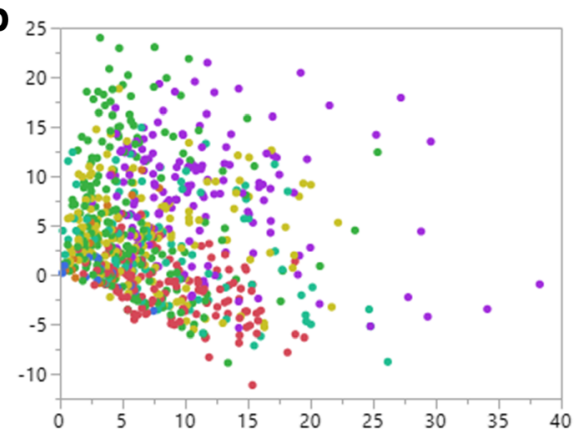

C

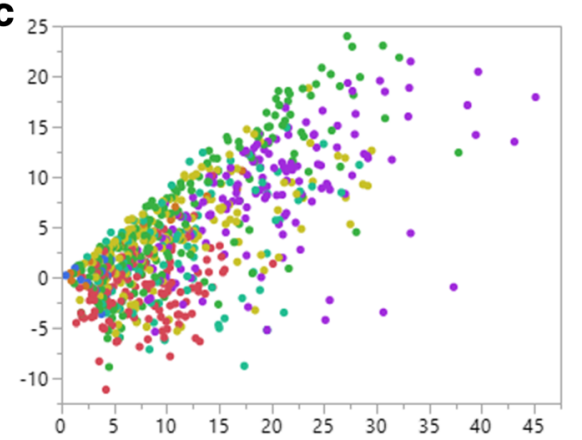

Fig. 1 Correlation of prevalence of wasting by weight-for-height, mid-upper arm circumference, and difference in prevalence. a Correlation of prevalence of global acute malnutrition (GAM) as determined by weight-for-height (Y-axis) and prevalence of GAM by mid-upper arm circumference (MUAC) (X-axis), by region. $\mathbf{b}$ Correlation of the difference in prevalence of GAM as determined by WHZ and prevalence of GAM by MUAC (Y-axis) and prevalence of GAM by MUAC (X-axis), by region. c Correlation of the difference in prevalence of GAM as determined by WHZ and prevalence of GAM by MUAC (Y-axis) and prevalence of GAM by WHZ (X-axis), by region. Regions represented by colors as follows: Latin America and the Caribbean (blue), Eastern and Southern Africa (green), Congo DRC (red), West and Central Africa (yellow), Middle East and North Africa (orange), South East Asia and Pacific (aqua), Sudan (purple)

were virtually unchanged when only surveys within $\pm 1.5 \%$ of the thresholds rather than $\pm 2.5 \%$ of the thresholds were included in the analysis: $4.46,7.06$, and $7.92 \%$, respectively. However, median wasting prevalence by MUAC corresponding to the famine threshold (30\%) was higher when only surveys within $\pm 1.5 \%$ of the thresholds were included (16.94\% vs. $15.71 \%)$; this estimate is likely less stable due to the smaller number of surveys in this threshold category.
Prevalence of wasting by MUAC for surveys with WHZ prevalence near all four thresholds varied considerably, as illustrated by the wide interquartile ranges and overall distributions (Fig. 2). For example, for surveys with wasting prevalence by WHZ approximately equal to $10 \%$, prevalence of wasting by MUAC ranged from less than $1 \%$ to nearly $20 \%$. The distributions for each of the four threshold categories all overlap substantially. Nearly half $(48.0 \%)$ of all surveys corresponding to the

Table 3 Parameter estimates from univariate and multivariate linear regression models of prevalence of wasting as assessed by mid-upper arm circumference

\begin{tabular}{|c|c|c|c|c|}
\hline \multirow[b]{2}{*}{ Dependent variables } & \multicolumn{2}{|l|}{ Univariate } & \multicolumn{2}{|l|}{ Multivariate } \\
\hline & Coefficient & $95 \% \mathrm{Cl}$ & Coefficient & $95 \% \mathrm{Cl}$ \\
\hline Intercept & 2.74 & $(2.14-3.34)^{\dagger+}$ & -9.36 & $(-15.03-3.70)$ \\
\hline Prevalence of Wasting, Weight-for-Height $<-2 Z$ & 0.43 & $(0.39-0.47)^{\dagger+}$ & 0.50 & $(0.46-0.54)^{+\dagger}$ \\
\hline Prevalence of Stunting, Height-for-Age $<-2 Z$ & & & 0.11 & $(0.09-0.12)^{\dagger \dagger}$ \\
\hline Percent of Children less than 30 Months of Age & & & 0.08 & $(0.03-0.14)^{\dagger+}$ \\
\hline Percent Females & & & 0.06 & $(-0.04-0.17)$ \\
\hline Model $R^{2}$ & 0.3623 & & 0.4560 & \\
\hline
\end{tabular}

${ }^{\dagger}$ Statistically significant, $p<0.05$

${ }^{++}$Statistically significant, $p<0.001$ 
Table 4 Median and interquartile range for prevalence of wasting by MUAC for surveys corresponding to existing nutritional crisis classification thresholds, by region

\begin{tabular}{|c|c|c|c|c|}
\hline & \multicolumn{4}{|c|}{ Prevalence of wasting by MUAC, [N] Median (IQR) } \\
\hline & Poor & Serious & Emergency & Famine \\
\hline & $5 \% \pm 2.5 \%$ & $10 \% \pm 2.5 \%$ & $15 \% \pm 2.5 \%$ & $30 \% \pm 2.5 \%$ \\
\hline Eastern and Southern Africa & [61] $4.10 \%(2.49-6.45)$ & [56] 4.41\% (2.89-6.96) & [18] 4.12\% (2.55-9.30) & [7] 9.64\% (7.55-14.95) \\
\hline Congo, DRC & [60] 5.66\% (4.46-7.99) & [46] $11.04 \%(7.75-14.30)$ & [17] 13.86\% (12.08-14.86) & \\
\hline West and Central Africa & [52] 3.35\% (1.85-5.61) & [42] 6.02\% (3.61-7.22) & [26] 8.29\% (5.86-10.28) & [3] $19.41 \%(16.88-20.02)$ \\
\hline Sudan & [2] $4.98 \%(4.51-5.46)$ & [27] 7.49\% (5.78-10.52) & {$[28] 7.89 \%(5.72-9.05)$} & [11] 16.48\% (11.70-17.50) \\
\hline South East Asia and Pacific & [21] 5.21\% (2.73-6.81) & [25] 7.03\% (4.64-11.85) & [20] 8.03\% (4.63-17.29) & [1] $17.13 \%$ \\
\hline \multirow[t]{2}{*}{ All Surveys ${ }^{a}$} & [211] 4.51\% (2.73-6.81) & {$[200] 6.67 \%(4.27-10.03)$} & {$[112] 8.15 \%(5.11-11.86)$} & [22] 15.71\% (10.28-17.50) \\
\hline & $5 \% \pm 1.5 \%$ & $10 \% \pm 1.5 \%$ & $15 \% \pm 1.5 \%$ & $30 \% \pm 1.5 \%$ \\
\hline Eastern and Southern Africa & [40] 4.04\% (2.47-7.29) & [31] 5.03\% (2.50-7.59) & [12] 3.62\% (2.35-9.31) & [2] 11.25\% (7.55-14.95) \\
\hline Congo, DRC & [37] 5.85\% (4.91-8.86) & [32] 11.22\% (8.25-13.88) & [11] 13.49\% (11.9-14.86) & \\
\hline West and Central Africa & [32] 3.53\% (1.91-5.61) & [25] 6.20\% (3.71-7.22) & [15] 8.12\% (5.56-8.78) & [3] 19.41\% (16.88-20.02) \\
\hline Sudan & [1] 5.46\% & [17] 7.11\% (5.78-9.91) & [15] 7.92\% (5.17-8.90) & [7] 17.00\% (12.32-19.72) \\
\hline South East Asia and Pacific & [15] 5.21\% (1.55-7.81) & [15] 7.03\% (4.64-11.04) & [10] 6.23\% (4.46-19.60) & \\
\hline All Surveys ${ }^{a}$ & [183] 4.46\% (2.58-7.25) & [122] 7.06\% (4.64-10.98) & [64] 7.92\% (4.46-11.99) & [12] 16.94\% (13.63-19.57) \\
\hline
\end{tabular}

a Surveys from Latin America and Caribbean region and the Middle East North Africa region are included among "All Surveys" but not presented separately by region given small numbers of surveys per category

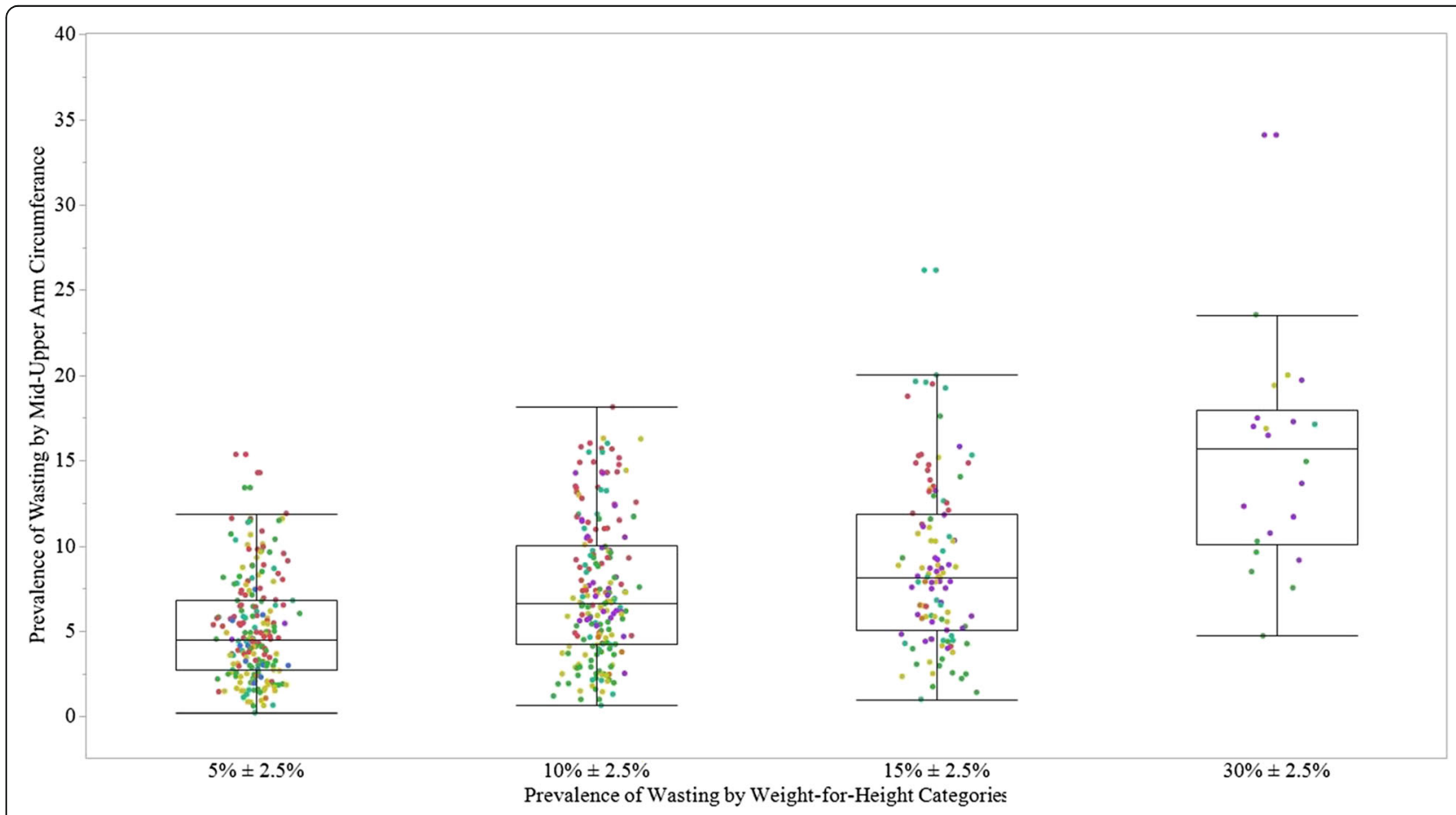

Fig. 2 Box-plots for prevalence of wasting by mid-upper arm circumference for surveys corresponding to existing crisis classification thresholds. Regions represented by colors as follows: Latin America and the Caribbean (blue), Eastern and Southern Africa (green), Congo DRC (red), West and Central Africa (yellow), Middle East and North Africa (orange), South East Asia and Pacific (aqua), Sudan (purple) 
$10 \%$ threshold $( \pm 2.5 \%)$, have prevalence of wasting within the IQR for the $15 \%$ threshold $( \pm 2.5 \%)$, too great an overlap to allow for meaningful discriminatory power.

Median prevalence of wasting by MUAC for each threshold category varied by region, suggesting that regional variation contributed to the overall variability observed. For surveys with prevalence of wasting by WHZ approximately equal to the 5, 10 and 15\% thresholds, median wasting prevalence by MUAC was greatest in the DRC. Median wasting by MUAC in DRC was nearly double that of surveys from West and Central Africa for surveys with wasting by WHZ of $5 \pm 2.5$ and $10 \% \pm 2.5 \%$ and more than triple that of surveys from Eastern and Southern Africa with wasting prevalence by WHZ of $15 \pm 2.5 \%$. Median prevalence of wasting by MUAC was also lowest in Eastern and Southern Africa for surveys near the famine threshold ( $30 \pm 2.5 \%)$.

Due to the large observed variation in wasting prevalence by MUAC corresponding to the current WHZ-based crisis thresholds, classification of surveys based on prevalence of MUAC and WHZ independently resulted in poor concordance regardless of the MUACbased thresholds used. Table 5 presents as illustration the proportion of surveys that would be classified into the same crisis category using a dozen MUAC threshold combinations derived based on analysis presented in Table 4 when compared with WHZ-categories of 5, 15 and $30 \%$. In all iterations, approximately 4 in 10 surveys were classified into the same crisis category. No combination of MUAC-based thresholds achieved greater than $50 \%$ concordance. Notably, Table 5 only contains suggested MUAC thresholds corresponding to 5, 15 and $30 \%$ WHZ thresholds. As shown in the previous analyses, the overlap in MUAC values around 10 and 15\% WHZ thresholds was too great to suggest a separate meaningful MUAC threshold for 10\% WHZ threshold. Including this threshold generally resulted in a lower proportion of concordant surveys.

\section{Discussion}

Analysis presented in this paper aimed to assess the feasibility of developing thresholds for determining the severity of a crisis in contexts where assessments of wasting using weight-for-height, the indicator for which WHO recommended emergency thresholds exist, were not practical and only mid-upper arm circumference could be measured. However, analysis of survey data from over 700 surveys from more than 40 countries suggests that prevalence of wasting as assessed by MUAC was poorly correlated with prevalence of wasting by WHZ. Correlation was not substantively improved when analysis was repeated separately by region; rho values for all regions were below 0.7. Consistent with previous literature [10], multivariable model demonstrated that an increase in prevalence of wasting by MUAC was significantly associated with an increased prevalence of stunting and an increased proportion of younger children (6 to 29 months of age) in the survey sample. Proportion of females in the sample was not significantly associated with the prevalence of wasting by MUAC. However, while prevalence of stunting and the proportion of younger children were both significant, including them in the model did not markedly improve fit $\left(\mathrm{R}^{2}\right.$ multivariate $=0.46 ; \mathrm{R}^{2}$ univariate $\left.=0.36\right)$. A poor correlation of wasting prevalence as assessed by WHZ and MUAC is consistent with previous literature on inconsistencies in diagnosis of individual children as wasted using WHZ and MUAC $[6,9,10]$.

Prevalence of wasting in most contexts was higher when assessed by WHZ than MUAC, however the reverse was true in approximately a quarter of all surveys.

Table 5 Classification into crisis categories surveys by prevalence of wasting by mid-upper arm circumference

\begin{tabular}{|c|c|c|c|c|c|}
\hline & \multicolumn{4}{|c|}{ Crisis categories based on prevalence of wasting by mid-upper arm circumference } & \multirow{2}{*}{$\begin{array}{l}\text { Percent } \\
\text { concordance } \\
(N=733)\end{array}$} \\
\hline & $\begin{array}{l}0-<5 \% \\
\text { Acceptable }\end{array}$ & $\begin{array}{l}5-<15 \% \\
\text { Poor/Serious }\end{array}$ & $\begin{array}{l}15-<30 \% \\
\text { Emergency }\end{array}$ & $\begin{array}{l}\geq 30 \% \\
\text { Famine }\end{array}$ & \\
\hline 1 & $<4 \%$ & $4-<7 \%$ & $7-<16 \%$ & $\geq 16 \%$ & $41.88 \%$ \\
\hline 2 & $<4 \%$ & $4-<7 \%$ & $7-<17 \%$ & $\geq 17 \%$ & $42.84 \%$ \\
\hline 3 & $<4 \%$ & $4-<8 \%$ & $8-<16 \%$ & $\geq 16 \%$ & $44.75 \%$ \\
\hline 4 & $<4 \%$ & $4-<8 \%$ & $8-<17 \%$ & $\geq 17 \%$ & $45.70 \%$ \\
\hline 5 & $<4 \%$ & $4-<9 \%$ & $9-<16 \%$ & $\geq 16 \%$ & $46.38 \%$ \\
\hline 6 & $<4 \%$ & $4-<9 \%$ & $9-<17 \%$ & $\geq 17 \%$ & $47.34 \%$ \\
\hline 7 & $<5 \%$ & $5-<7 \%$ & $7-<16 \%$ & $\geq 16 \%$ & $37.93 \%$ \\
\hline 8 & $<5 \%$ & $5-<7 \%$ & $7-<17 \%$ & $\geq 17 \%$ & $38.88 \%$ \\
\hline 9 & $<5 \%$ & $5-<8 \%$ & $8-<16 \%$ & $\geq 16 \%$ & $40.79 \%$ \\
\hline 10 & $<5 \%$ & $5-<8 \%$ & $8-<17 \%$ & $\geq 17 \%$ & $41.75 \%$ \\
\hline 11 & $<5 \%$ & $5-<9 \%$ & $9-<16 \%$ & $\geq 16 \%$ & $42.43 \%$ \\
\hline 12 & $<5 \%$ & $5-<9 \%$ & $9-<17 \%$ & $\geq 17 \%$ & $43.38 \%$ \\
\hline
\end{tabular}


The difference in prevalence of wasting by WHZ and MUAC varied considerably, even within the same country and region. Interestingly, our analysis suggest that this difference in prevalence was more strongly correlated with prevalence of wasting by WHZ $(\rho=0.69)$ than the correlation of the two prevalence estimates directly $(\rho=0.55)$. On the other hand, this difference in prevalence was poorly correlated with the prevalence of wasting by MUAC $(\rho=-0.16)$. Given the main focus of this analysis on predicting prevalence of wasting by WHZ in contexts where only wasting by MUAC is known, the high correlation of wasting by $\mathrm{WHZ}$ and the difference in wasting prevalence has limited practical utility; wasting by MUAC has very little predictive power on the difference between the prevalence by WHZ and MUAC.

Developing an algorithm for conversion between prevalence of wasting by MUAC and WHZ, or converting WHZ-based thresholds to MUAC-based thresholds, is inadvisable given the observed correlation and high heteroscedasticity. Previous research deriving formulas for converting between prevalence estimates have been based on much stronger correlations. For example, the algorithm to convert from estimates of child malnutrition using the National Center for Health Statistics (NCHS) growth reference to estimates using the new WHO growth standards was based on a very high degree of fit $\left(R^{2}>0.9\right)$ of the regression models used to derive conversion formulas; $\mathrm{R}^{2}$ for wasting model was 0.96 [15].

The follow-up analysis presented in Tables 4 and 5 and Fig. 2 illustrates how poor correlation between prevalence of wasting by WHZ and MUAC translates into frequent discordant classification using MUAC- and WHZ-based thresholds. For surveys with prevalence of wasting by WHZ within $\pm 1.5 \%$ of each of the crisis thresholds, the prevalence of wasting by MUAC varied by $15 \%$ points or more for each threshold. The box plots for wasting prevalence by MUAC for surveys near thresholds of 10 and $15 \%$ as assessed by WHZ overlapped almost entirely. Even when serious and emergency categories were combined, no combination of thresholds resulted in concordant phase classification of wasting by WHZ and MUAC for more than half of the surveys. Further, the data do not support the development of region-specific thresholds. While median prevalence of wasting by MUAC for surveys within $\pm 1.5 \%$ of each of the crisis thresholds did vary by region, within regions the variability of prevalence around the median remained high. Findings on poor correlation between prevalence of wasting by WHZ and MUAC confirm that MUAC-based thresholds cannot be derived from WHZbased thresholds. They also highlight the broader issue of the lack of evidence that underlies the indicators currently used to classify severity of emergencies. Further research would be needed to develop MUAC-based thresholds that are independent of the current WHZbased thresholds and objectively define crisis severity, potentially considering functional outcomes (e.g., morbidity, mortality), and response to treatment.

This analysis is subject to several limitations. First, included surveys disproportionately represent countries with refugees, displaced persons, and/or experiencing chronic nutrition emergencies-Democratic Republic of Congo, Sudan, Chad, Ethiopia, Kenya. Surveys were all conducted by one of two agencies (ACF and UNHCR). This analysis includes only surveys made available for analysis. If repeated on a different set of surveys, this analysis may yield slightly different estimates, however the overall pattern of relationship is not likely to change. Second, the dataset contains relatively few surveys with very high prevalence (near famine thresholds) reflecting the fact that these contexts are fortunately relatively rare. Estimates of wasting prevalence by MUAC corresponding with high wasting prevalence by WHZ are therefore less stable compared with estimates for lower prevalence thresholds. Finally, while available data allowed for adjustment in regression analysis for key predictors of MUAC such as stunting, age and sex distribution, data on sitting-to-standing height ratio, another moderately important predictor of wasting by MUAC diagnosis was not measured in the surveys [10].

\section{Conclusion}

In summary, estimates of wasting from MUAC-only assessments, such as those collected in humanitarian contexts with great insecurity, cannot be reliably converted to estimates of wasting by WHZ as prevalence of wasting assessed by MUAC is poorly correlated with prevalence wasting assessed by WHZ. As such, data presented in this analysis does not support the development of MUACbased crisis thresholds corresponding with the current WHZ-based thresholds. In addition, applying current WHZ-based thresholds $(5,10,15,30 \%)$ to prevalence of wasting assessed by MUAC is not recommended, especially in contexts with high prevalence of wasting by WHZ. As demonstrated, the difference in prevalence of wasting as assessed by WHZ and by MUAC is greatest in crises when wasting becomes more prevalent, so the estimates from MUAC-only assessments will tend to provide lower estimates of crisis severity than WHZ.

\section{Abbreviations \\ ACF: Action Contre la Faim; CH: Cadre Harmonisé; DRC: Democratic Republic of Congo; IFRC: International Federation of Red Cross; IPC: Integrated Phase Classification; IQR: Interquartile range; MUAC: Mid-upper arm circumference; SCN: Standing Committee on Nutrition; UNHCR: United Nations High Commissioner for Refugees; UNICEF: United Nations Children's Fund; WHO: World Health Organization; WHZ: Weight-for-height Z scores}

\section{Acknowledgements}

Erin Hulland, CDC; Curtis Blanton, CDC; Rebecca Wee, CDC; Action Contre la Faim (ACF); United Nations High Commissioner for Human Rights (UNHCR). 


\section{Availability of data and materials}

The data that support the findings of this study are available from Action Contre la Faim and the United Nations High Commissioner for Human Rights but restrictions apply to the availability of these data, which were used under license for the current study, and so are not publicly available. Data are however available from the authors upon reasonable request and with permission of Action Contre la Faim and the United Nations High Commissioner for Human Rights.

\section{Authors' contributions}

Both authors contributed equally to this study. EL, OB designed the study, $\mathrm{EL}, \mathrm{OB}$ collected the data, EL, OB analyzed and interpreted the data, EL and $\mathrm{OB}$ drafted the manuscript, EL, OB critically revised the manuscript for important intellectual content. EL, OB read and approved the final manuscript. EL is a guarantor. Both authors have read and approved the manuscript, have full access to all of the data, and take responsibility for the integrity of the data and the accuracy of the data analysis.

\section{Ethics approval and consent to participate}

Not applicable. Analysis based on secondary analysis of anonymized data where no individual data could be identified so formal ethical clearance was not required. Permission to use the data were obtained from the organizations collecting the datasets.

The findings and conclusions in this report are those of the authors and do not necessarily represent the official position of the Centers for Disease Control. UNHCR data is part of the Public Health Dataset. UNHCR does not warrant in any way the accuracy of the data or information reproduced from the provided Public Health Dataset and may not be held liable for any loss caused by reliance on the accuracy or reliability thereof.

\section{Competing interests}

All authors declare that they have no competing interests; no support from any organization for the submitted work; no financial relationships with any organizations that might have an interest in the submitted work in the previous 3 years; no other relationships or activities that could appear to have influenced the submitted work.

\section{Publisher's Note}

Springer Nature remains neutral with regard to jurisdictional claims in published maps and institutional affiliations.

Received: 13 September 2017 Accepted: 8 May 2018

Published online: 18 May 2018

\section{References}

1. World Health Organization. Physical status: the use and interpretation of anthropometry. Geneva, Switzerland: Report of a WHO Expert Committee; 1995.

2. World Health Organization. The management of nutrition in major emergencies. Geneva, Switzerland: World Health Organization; 2000.

3. Howe P, Devereux S. Famine intensity and magnitude scales: a proposal for an instrumental definition of famine. Disasters. 2004;28(4):353-72.

4. Cadre Harmonise. Identification and Analysis of Areas at Risk and Populations Affected by Food and Nutrition Insecurity in the Sahel and West Africa: Manual. Niamey, Niger; 2016. Available at: http://fscluster.org/ nigeria/document/cadre-harmonise-manual-version-10.

5. Integrated Food Security Phase Classification Global Partners. Technical manual version 2.0: evidence and standards for better food security decisions. Rome, Italy: Food and Agriculture Organization; 2012. Available at: http://www.ipcinfo.org/fileadmin/user_upload/ipcinfo/docs/IPC-Manual-2Interactive.pdf.

6. World Health Organization, United Nations Children's Fund. WHO child growth standards and the identification of severe acute malnutrition in infants and children. Geneva, Switzerland: A Joint Statement; 2009.

7. World Health Organization, United Nations Children's Fund, Standing Committee on Nutrition. Informal consultation on community-based management of severe malnutrition in children. Geneva, Switzerland: SCN Nutrition Policy; 2006.

8. World Health Organization, United Nations Children's Fund, World Food Programme, Standing Committee on Nutrition. Joint Statement. Community-based management of severe acute malnutrition. Geneva, New York, Rome; 2007.
9. Grellety EG, Golden MH. Weight-for-height and mid-upper-arm circumference should be used independently to diagnose acute malnutrition: policy implications. BMC Nutrition. 2016;2:10.

10. Roberfroid D, Huybregts L, Lachat C, Vrijens F, Kolsteren P, Guesdon B. Inconsistent diagnosis of acute malnutrition by weight-for-height and mid-upper arm circumference: contributors in 16 cross-sectional surveys from South Sudan, the Philippines, Chad, and Bangladesh. Nutr J. 2015;14:86.

11. Briend A, Mwangome M, Berkley J. (2017). Using Mid-Upper Arm Circumference to Detect High-Risk Malnourished Patients in Need of Treatment. In Preedy VR, Patel VB (Eds.) Handbook of Famine, Starvation, and Nutrient Deprivation (pp 1-17). Springer international Publishing.

12. Action Contre la Faim. Anthropometric survey data, 2001-2013. Paris: Action Contre la Faim International; 2015.

13. United Nations High Commissioner for Refugees. Standardised expanded nutrition survey, 2011-2016. Geneva: UNHCR; 2017.

14. WHO Multicentre Growth Reference Group. Child growth standards: length/ height-for-age, weight-for-age, weight-for-length, weight-for-height and body mass index-for-age: methods and development. Geneva, Switzerland: World Health Organization; 2006.

15. Yang $\mathrm{H}$, de Onis $\mathrm{M}$. Algorithms for converting estimates of child malnutrition based on the NCHS reference into estimates based on the WHO child growth standards. BMC Pediatr. 2008;8:19.

\section{Ready to submit your research? Choose BMC and benefit from:}

- fast, convenient online submission

- thorough peer review by experienced researchers in your field

- rapid publication on acceptance

- support for research data, including large and complex data types

- gold Open Access which fosters wider collaboration and increased citations

- maximum visibility for your research: over $100 \mathrm{M}$ website views per year

At BMC, research is always in progress.

Learn more biomedcentral.com/submissions 